Surgery Journal 5 (2): 17-21, 2010

ISSN: $1816-3211$

(C) Medwell Journals, 2010

\title{
Local and Systemic TNF- $\alpha$ Level Correlation to Histopathology in Post-Mastectomy Seroma in Rabbits
}

\author{
${ }^{1}$ Z.K. Al-Gaithy, ${ }^{2}$ S.S. Ali, ${ }^{2,}{ }^{3}$ N.N. Ayuob and ${ }^{4}$ N. Al-Sawy \\ ${ }^{1}$ Department of Surgery, Faculty of Medicine, \\ ${ }^{2}$ Department of Anatomy, Faculty of Medicine, \\ King Abdul Aziz University, Kingdom of Saudi Arabia \\ ${ }^{3}$ Department of Histology and Cytology, Faculty of Medicine, Mansoura University, \\ Mansoura, Dakahliya, Egypt \\ ${ }^{4}$ Department of Biochemistry, Faculty of Science, \\ King Abdul Aziz University, Kingdom of Saudi Arabia
}

\begin{abstract}
Seroma is a common problem after mastectomy, occurring in 30-92\% of cases. The aim of this study was to detect the histopathology of postmastectomy seromas and the related changes in tumor necrosis factor- $\alpha(\mathrm{TNF}-\alpha)$ serum levels. Unilateral mastectomies were performed on 45 female albino rabbits. On day 7 , the area of mastectomy was palpated for the presence of swelling and any fluid was aspirated. A biochemical assay of TNF- $\alpha$ serum from all animals (with and without seroma) and that found in the aspirated fluid was performed. Skin flaps and underlying tissues of the mastectomy region were dissected, fixed in $10 \%$ formalin, processed and stained for histopathological examination. Seroma developed in $46.6 \%$ of the rabbits. The mean volume of seroma fluids was $3.03 \pm 2.21 \mathrm{~mL}$. The level of TNF- $\alpha$ in the serum of rabbits that developed seroma was significantly higher than in those that did not. The tissue surrounding the seromas showed persistent granulation tissue containing fibroblasts, inflammatory cells and a large number of newly formed blood vessels surrounded by extravasated blood. This could be attributed to the increased TNF- $\alpha$ serum level, as discovered by the biochemical study. The inflammatory cell infiltrates mainly consisted of eosinophils as well as lymphocytes, macrophages and plasma cells. The postmastectomy seromas showed altered and delayed wound healing. TNF- $\alpha$ levels were elevated in both the seroma fluid and the serum of animals that developed seroma, possibly indicating its role in seroma development.
\end{abstract}

Key words: Inflammatory, extravasted blood, plasma cells, biochemical, lymohocytes, seroma

\section{INTRODUCTION}

Seroma refers to any collection of serous, sterile fluid within a fibrous pseudomembrane (dead space) under skin incisions or around artificial vascular grafts. It is a common problem after mastectomy, occurring in 30-92\% of cases and often continues after removal of the suction drain. Thus, repeated skin puncturing is necessary to remove the seroma (Axelsson, 2007).

Modified radical mastectomy with axillary lymph node dissection is a common procedure to treat breast cancer. Seroma is the most common wound complication after modified radical mastectomy, occurring in approximately $50 \%$ of patients undergoing mastectomy (Stehbens, 2003; Say and Donegan, 1974). Many patient factors may increase or decrease seroma formation (Pogson et al.,
2003; Kuroi et al., 2006). It might also be operator-dependent and related to surgical techniques (Kuroi et al., 2006). Although, seromas usually vanish within a few weeks, some patients may require repeated aspirations even for a period of months. Prolonged accumulation of postmastectomy seroma and repeated aspirations predisposes patients to sepsis and other wound-related complications and may delay adjuvant therapy.

Tumor necrosis factor- $\alpha$ (TNF- $\alpha)$ is released by the leukocytes extravasating out of blood vessels and is crucial in initiating, controlling and terminating the cellular events of wound healing. It has been found that TNF- $\alpha$ may also be associated with the wound healing processes, especially in the later phases and has been shown to induce angiogenesis. It also promotes the

Corresponding Author: N.N. Ayuob, Department of Histology and Cytology, Faculty of Medicine, Mansoura University, Mansoura, Dakahliya, Egypt 
synthesis of collagenase and prostaglandin E2 in fibroblasts. These properties may be important for the formation of granulation tissue and the remodeling of the wound matrix (Fu et al., 1996).

The aim of this study was to detect the histopathological changes of postmastectomy seromas as well as related changes in TNF- $\alpha$ serum levels.

\section{MATERIALS AND METHODS}

The study protocol was approved by the Biomedical Research Ethics Committee of King Abdul Aziz Medical College. About 45 female albino rabbits (3-4 months old) weighing between $2.5-4.5 \mathrm{~kg}$ were used. All animals were fed standard food supplied by the animal care unit of King Fahed Medical Research Center at King Abdul Aziz University. The rabbits were administered ketamine (50 $\mathrm{mg} \mathrm{mL}^{-1}$ ) (Especialidad USO Hospital Ario) and Seton (2\%) (Especialidad USO Veterinario). Complete aseptic procedures were ensured before the operation. Unilateral mastectomies were performed by modifying the method used for rats by other researchers (Lindsey et al., 1990). An elliptical vertical incision was made around the nipple of the pectoral breast. The nipple with the surrounding skin was discarded.

A visual inspection of all operated animals was performed on a daily basis after the procedure, checking arm movements and wound complications such as wound erythema, dehiscence, infection and swelling. On day 7 , the areas of mastectomy were inspected and palpated for the presence of swelling.

For those animals with seromas, the fluid was aspirated from the most dependent axillary region using a syringe. The dead space was opened and any remaining fluid was aspirated. The aspirated fluid was inspected for any turbidity, blood staining or suspended particles. The fluid was centrifuged and the supernatant was kept at $-70^{\circ} \mathrm{C}$ before being analyzed for (TNF- $\alpha$ ) cytokines. Biochemical assays of TNF- $\alpha$ in the serum of both seroma and non-seroma animals as well the seroma fluid itself were performed in the biochemistry lab at King Abdul Aziz University Hospital. An Enzyme-Linked Immunosorbent Assay (ELISA) was carried out for the quantitative detection of rabbit TNF- $\alpha$.

After fluid aspiration, the opened post-mastectomy dead space was inspected and photographed. Skin flaps and underlying tissues of the mastectomy region including the axilla and thoracic wall were dissected and fixed in $10 \%$ formalin. The tissues were trimmed to include the dead space floor, the overlying skin, parts of the underlying chest muscles and axillary fat. Paraffin sections of a thickness of $5 \mu$ were stained by Haematoxylin, Eosin ( $\mathrm{Hx}$ and $\mathrm{E}$ ) and Mallory trichrome for histological examination.

\section{RESULTS AND DISCUSSION}

Seroma formation: The wound incision started to close on the 3rd day after the mastectomy and was almost complete by the 7 th day. About 15 rabbits were excluded due to postoperative death, hematomas and infection. Those that developed seromas showed raised skin and a cystic swelling containing fluid underneath the skin flap. Seromas developed in 14 rabbits $(46.6 \%)$. In most of those cases, the seroma fluid was clear and strawberry in color (serous or serosanginous). It did not clot on standing. The mean volume of seroma fluids was $3.03 \pm 2.21 \mathrm{~mL}$.

Biochemical findings: The results of biochemical analysis of total protein, sodium, potassium and chloride concentrations of seroma fluid are shown in Table 1. The mean level of TNF- $\alpha$ in the serum of rabbits that developed seroma and those that not was $10.31 \pm 0.5$ and $8.85 \pm 0.69$, respectively. The level of TNF- $\alpha$ in the serum of rabbits that developed seroma was significantly higher than in those that did not. The level of TNF- $\alpha$ in the seroma fluid of rabbits that developed seroma was $5.72 \pm 0.74$ (Table 2).

Histopathological findings: A histopathological examination of the postmastectomy regions in the seroma group showed a large dead space with a ragged floor and defective fibrous wall formation. The reformed epithelium was defective and the underlying muscles appeared degenerated (Fig. 1). The tissue surrounding the seromas showed persistent granulation tissue containing fibroblasts, some inflammatory cells and a large number of newly formed blood vessels. The latter were surrounded by a large amount of extravasated blood suggesting that the vessel walls were defective (Fig. 2a, b). The inflammatory cell infiltrates contained a large number of eosinophils and other cells such as lymphocytes, macrophages and plasma cells (Fig. 2c, d).

Table 1: Levels of Total Protein (TP), sodium, potassium and chloride concentration in seroma fluid

\begin{tabular}{lllll} 
Results & $\mathrm{Na}\left(\mathrm{mmol} \mathrm{L}^{-1}\right)$ & $\mathrm{K}\left(\mathrm{mmol} \mathrm{L}^{-1}\right)$ & $\mathrm{CL}\left(\mathrm{mmol} \mathrm{L}^{-1}\right)$ & $\mathrm{TP}\left(\mathrm{g} \mathrm{L}^{-1}\right)$ \\
\hline
\end{tabular} \begin{tabular}{lllll}
\hline Mean \pm SD & $139.57 \pm 2.88$ & $5.11 \pm 0.95$ & $105.71 \pm 3.77$ & $44.86 \pm 6.82$
\end{tabular}

Range $\quad(134.00-142.00) \quad(4.10-6.90) \quad(102.00-113.00) \quad(36.00-53.00)$

Table 2: Serum TNF- $\alpha$ level in rabbits developed Seroma and those not develop seroma

TNF- $\alpha$ level in Serum TNF- $\alpha$ of $\quad$ Serum TNF- $\alpha$ of
seroma fluid none seroma group seroma group

Results $\left.\quad\left(\mathrm{pg} \mathrm{mL}^{-1}\right) \mathrm{N}=14 \quad\left(\mathrm{pg} \mathrm{mL}^{-1}\right) \mathrm{N}=14 \quad(\mathrm{pg} \mathrm{mL})^{-1}\right) \mathrm{N}=14$

\begin{tabular}{llll}
\hline Mean \pm SD & $5.72 \pm 0.74$ & $8.85 \pm 0.69$ & $10.31 \pm 0.5^{*}$ \\
\hline
\end{tabular}

$\mathrm{N}=$ Number of cases; "Significant difference; $\mathrm{p}>0.05$ 


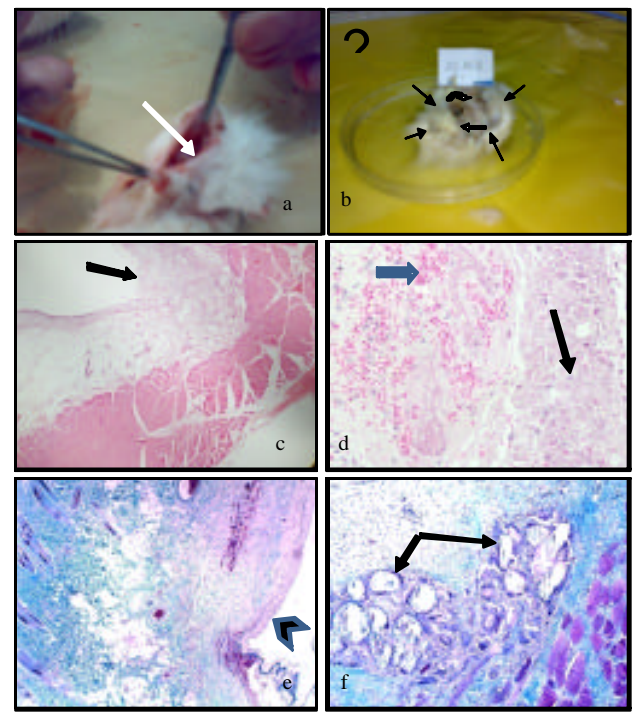

Fig. 1: Photographs to show the site of post mastectomy suture at 7 th day, the dead space was found to be narrow with smooth floor (white arrow). Photograph of seroma tissue (black arrows). It showed the exacerbate granulation tissue (white arrow). Notice the presence of necrotic brownish tissue (curved white arrow) at one part of the floor. Photomicrographs of part of seroma developed in post mastectomy region. There was no fibrous wall (thick black arrow). The epithelium was defectively reformed (arrow head). The underlying muscles appeared degenerated (thin black arrow). Note the extravasated blood inbetween muscles (white arrow) (AX100 and BX200 H and E, C X100 and DX200 Mallory trichrome)

The postmastectomy regions in rabbits that did not develop seromas showed a small dead space. The seroma floors were well delineated by flat, close endothelial-like cells. The underlying tissue showed an almost mature fibrous scar containing active fibroblasts with no signs of vascular dilation or congestion. No inflammatory cell infiltrates were observed and the overlying skin was healed (Fig. 2e, f).

Seroma formation frequently occurs in patients who undergo axillary lymphadenectomy which is an integral part of breast cancer treatment. Seromas occur in 15-85\% of these cases (Ruggiero et al., 2009; Cipolla et al., 2010) and are challenging postoperative complications of unclear origin that are often spontaneously resolved. Seromas are of marked interest in terms of both pathogenesis and management (Lumachi et al., 2004; Nadkarni et al., 2007).

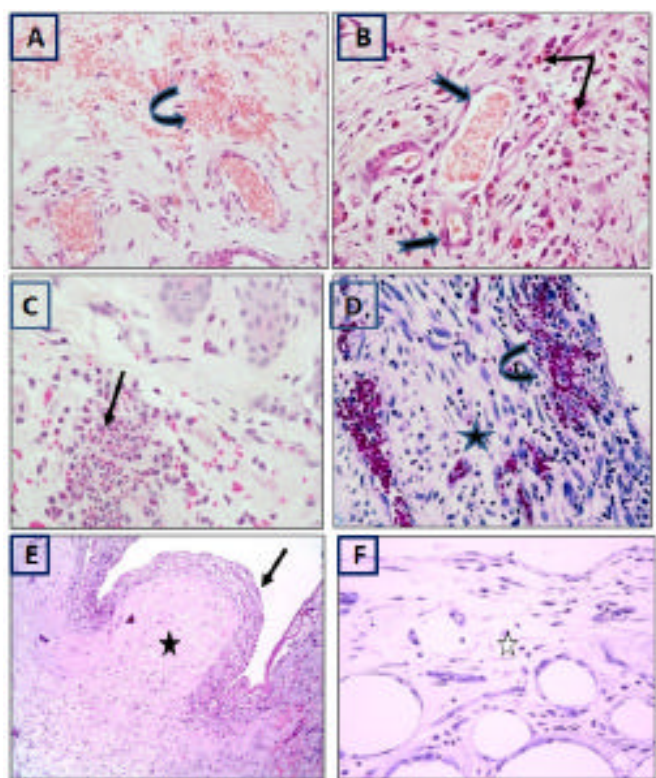

Fig. 2: Seroma bed showed persistence of the granulation tissue (star) and many newly formed blood vessels (Black bifid arrow) surrounded by extravasated blood (curved arrow). Thin black arrows indicated the esinophils (AX200 $\mathrm{H}$ and $\mathrm{E}$, BX400, CX200, H and E and DX200 Mallory trichrom). Showed post mastectomy regions in animals without seroma. The floor lined by endothelial cells (black arrow). Mature fibrous tissue contained inactive fibroblasts (white star). No vascular congestion or inflammatory cell infiltrate detected in the underling tissues (black star) (EX200 and FX400 H and E)

However, research about the histopathological changes of seromas are lacking thus, this study was undertaken. This study assessed the seroma-related changes of one of the cytokines, TNF- $\alpha$, involved in the wound healing process.

In this study, seromas developed in $46.6 \%$ of the rabbits that underwent unilateral mastectomies with nodal dissection. This is consistent with the results reported by Dawson et al. (1989) and Beamer et al. (2000). Biochemical examination of the fluid showed the seromas were compositionally different from lymph fluid, yet were similar to inflammatory exudates. Thus, seromas may be considered to be a result of a prolonged inflammatory phase of wound healing. Thus, anti-inflammatory drugs or chemicals that show local anti-inflammatory properties may diminish abundant fluid accumulation at surgical wound sites (Bonnema et al., 1999).

Chow found that after surgery, IL- 6 and c-reactive protein serum levels were elevated while $\mathrm{TNF}-\alpha$ serum 
levels were diminished. This study showed that the level of TNF- $\alpha$ in the serum of rabbits that developed seromas was significantly higher than in rabbits that did not. This indicates that the excess of TNF- $\alpha$ in the serum and hence, in the tissue fluids at the site of the wound led to a delay in the wound healing process. This would result in chronic unhealed wounds or complications of the healing process, e.g., flap necrosis or seroma formation. This explanation was postulated by Gallucci et al. (2000). TNF- $\alpha$ has also been shown to induce angiogenesis (Fu et al., 1996) which could explain the presence of excess newly formed blood vessels observed upon histopathological examination. Elevated TNF- $\alpha$ serum levels and the proven role in inducing angiogenesis could explain the fluid accumulation in seromas as a result of leaking newly formed blood vessels. This adverse effect of high TNF- $\alpha$ levels can be blocked by a specific TNF- $\alpha$ antagonist (TNF- $\alpha$ binding protein) (Maish et al., 1998).

A histological examination of postmastectomy regions in the seroma group showed a large dead space with a ragged floor and defective fibrous wall formation.

The reformed epithelium was also defective. This finding is in partial agreement with some researchers who reported a case of persistent seroma following modified radical mastectomy with fibrous tissue capsules and predominantly lymphocytic infiltration (Stanczyk et al., 2007). This study showed that the postmastectomy seroma regions contained large amounts of inflammatory infiltrates, consisting mostly of eosinophils. This finding is in partial agreement with some researchers who investigated the biochemical and hematological features of postmastectomy seromas (McCaul et al., 2000). They showed that seromas contain significantly more granulocytes and monocytes than lymphocytes.

The predominating eosinophils in the present samples had a role in vascular dilatation and fluid efflux. This can possibly be mediated by histamine, the chemical mediator released by eosinophils which has been reported to have a role in seroma formation (Wu et al., 2003). It has been suggested that vasodilation is obvious during the inflammation period of wound healing and is a crucial event for cellular efflux and fluid extravasation (Stehbens, 2003, Lorenz and Longaker, 2001).

The leukocytes extravasating from the blood vessels formed the infiltrates seen in the surrounding tissue of the seromas. These released cytokines during the wound healing process, something that has been recorded by many researchers (Wood et al., 1997; Lin et al., 2000; Calvano et al., 2000). These cytokines such as interleukin 6 (IL-6) and TNF- $\alpha$ are crucial in initiating, controlling and terminating the cellular events of wound healing. These cellular events are responsible for the degradation of the extracellular matrix, the migration and proliferation of cells, angiogenesis and matrix remodeling (Baker and Leaper, 2000).

\section{ACKNOWLEDGEMENT}

The researchers thank the Deanship of the Scientific Research for funding this research project (number 429/019-5).

\section{REFERENCES}

Axelsson, C., 2007. Does a single steroid injection reduce the formation of postmastectomy seroma. NCT00307606-Herlev-000 (Clinical Trial 139124). http://www. clinicalconnection.com/exp/ExpandedP atientViewStudy139124.aspx.

Baker, E.A. and D.J. Leaper, 2000. Proteinases, their inhibitors, and cytokine profiles in acute wound fluid. Wound Repair Regenerat., 8: 392-398.

Beamer, S.D., M.F. Helmer, M. Boyle and P.A. Woodworth, 2000. Seroma formation after breast cancer surgery: Incidence and predicting factors. Am. Surgeon, 66: 444-450.

Bonnema, J., D.A. Ligtenstein, T. Wiggers and A.N. van Geel, 1999. The composition of serous fluid after axillary dissection. Eur J. Surg., 165: 9-13.

Cipolla, C., S. Fricano, S. Vieni, G. Graceffa, G. Licari, A. Torcivia and M.A. Latteri, 2010. Does the use of fibrin glue prevent seroma formation after axillary lymphadenectomy for breast cancer: A prospective randomized trial in 159 patients. J. Surg. Oncol., 101: 600-603.

Dawson, I., L. Stam, J.M. Heslinga and H.L. Kalsbeek, 1989. Effect of shoulder immobilization on wound seroma and shoulder dysfunction following modified radical mastectomy: A randomized prospective clinical trial. Br. J. Surg., 76: 311-312.

Fu, X., H. Tian, S. Hsu, D. Wang and Z. Sheng, 1996. In vivo effects of tumor necrosis factor-alpha on incised wound and gunshot wound healing. J. Trauma., 40: S140-S1 43.

Gallucci, R.M., P.P. Simeonova, J.M. Matheson, C. Kommineni, J.L. Guriel, T. Sugawara and M.I. Luster, 2000. Impaired cutaneous wound healing in interleukin-6-deficient and immunosuppressed mice. Federation Am. Soc. Exp. Biol., 14: 2525-2531.

Kuroi, K., K. Shimozuma, T. Taguchi, H. Imai, H. Yamashiro, S. Ohsumi and S. Saito, 2006. Effect of mechanical closure of dead space on seroma formation after breast surgery. Breast Cancer, 13: $260-265$. 
Lin, E., S.E. Calvano and S.F. Lowry, 2000. Inflammatory cytokines and cell response in surgery. Surgery, 127: 117-126.

Lindsey, W.H., T.M. Masterson, W.D. Spotnitz, M.C. Wilhelm and R.F. Morgan, 1990. Seroma prevention using fibrin glue in a rate mastectomy model. Arch Surg., 125: 305-307.

Lorenz, H.P. and M.T. Longaker, 2001. Wounds: Biology, Pathology and Management. In: Surgery: Basic Science and Clinical Evidence, Norton, J.A., R.R. Bollinger, A.E. Chang, S.F. Lowry and S.J. Mulvihill et al. (Eds.). Springer-Verlag, New York, pp: 77-88.

Lumachi, F., A.A. Brandes, P. Burelli, S.M. Basso, M. Iacobone and M. Ermani, 2004. Seroma prevention following axillary dissection in patients with breast cancer by using ultrasound scissors: A prospective clinical study. Eur. J. Surg. Oncol., 30: 526-530.

Maish, G.O., M.L. Shumate, H.P. Ehrlich and R.N. Cooney, 1998. Tumor necrosis factor binding protein improves incision wound healing in sepsis. J. Surg. Res., 78: 108-117.

McCaul, J.A., A. Aslaam, R.J. Spooner, I. Louden, T. Cavanagh and A.D. Purushotham, 2000. Aetiology of seroma formation in patients undergoing surgery for breast cancer. Breast, 9: 144-148.
Nadkarni, M.S., A.K. Rangole, R.K. Sharma, R.V. Hawaldar, V.V. Parmar and R.A. Badwe, 2007. Influence of surgical technique on axillary seroma formation: A randomized study. ANZ J. Surg., 77: 385-389.

Pogson, C.J., A. Adwani and S.R. Ebbs, 2003. Seroma following breast cancer surgery. EJSO., 29: 711-717.

Ruggiero, R., E. Procaccini, S. Gili, C. Cremone and D. Parmeggiani et al., 2009. New trends on fibrin glue in seroma after axillary lymphadenectomy for breast cancer. G. Chir., 30: 306-310.

Say, C.C. and W. Donegan, 1974. Biostatistical evaluation of complications from mastectomy. Surg. Gynaecol. Obstet., 138: 370-376.

Stanczyk, M., B. Grala, T. Zwierowicz and M. Maruszynski, 2007. Surgical resection for persistent seroma, following modified radical mastectomy. World J. Surg. Oncol., 5: 104-104.

Stehbens, W.E., 2003. Postmastectomy serous drainage and seroma: Probable pathogenesis and prevention. ANZ J. Surg., 73: 877-880.

Wood, F., T.A. Griffiths and M. Stoner, 1997. Epidermalderived factors in the treatment of a chronic leg ulcer. J. Wound Care, 6: 256-258.

Wu, F.P.K., K. Hoekman, S. Meijer and M.A. Cuesta, 2003. VEGF and endostatin levels in wound fluid and plasma after breast surgery. Angiogenesis, 6: 255-257. 